Documentation et bibliothèques

DOCUMENTATION BIBLIOTHËQUES

\title{
Les gens d'affaires à la bibliothèque publique, une réalité et un succès! L'exemple du Centre d'affaires.doc de la Bibliothèque de Brossard
}

\section{Julie Desautels}

Volume 57, numéro 3, juillet-septembre 2011

URI : https://id.erudit.org/iderudit/1028844ar

DOI : https://doi.org/10.7202/1028844ar

Aller au sommaire du numéro

Éditeur(s)

Association pour l'avancement des sciences et des techniques de la documentation (ASTED)

\section{ISSN}

0315-2340 (imprimé)

2291-8949 (numérique)

Découvrir la revue

Citer cet article

Desautels, J. (2011). Les gens d'affaires à la bibliothèque publique, une réalité et un succès! L'exemple du Centre d'affaires.doc de la Bibliothèque de Brossard. Documentation et bibliothèques, 57(3), 178-180.

https://doi.org/10.7202/1028844ar

Tous droits réservés (C) Association pour l'avancement des sciences et des techniques de la documentation (ASTED), 2011
Ce document est protégé par la loi sur le droit d'auteur. L'utilisation des services d'Érudit (y compris la reproduction) est assujettie à sa politique d'utilisation que vous pouvez consulter en ligne.

https://apropos.erudit.org/fr/usagers/politique-dutilisation/ 


\section{Les gens d'affaires à la bibliothèque publique, une réalité et un succès ! Lexemple du Centre d'affaires.doc de la Bibliothèque de Brossard}

\section{JULIE DESAUTELS}

Bibliothécaire professionnelle Responsable du Centre d'affaires.doc

\section{$\frac{\text { Centre J'affaires }}{. \mathrm{dOC}}$}

\section{Présentation}

$\mathrm{E}$ N 1999, LA BibliothèQue DE BRossard faisait preuve de dynamisme et d'innovation par la création du Centre d'affaires.doc ${ }^{1}$ en ses lieux. En effet, elle a été la première bibliothèque de la RiveSud à offrir un service à la communauté d'affaires afin d'appuyer les initiatives d'animation économique de la Ville de Brossard. En ce sens, le Centre a contribué et contribue toujours au développement économique local et régional en fournissant aux entrepreneurs et travailleurs autonomes de l'information stratégique essentielle au développement de leurs activités dans un contexte économique compétitif.

Le Centre est un lieu de recherche, de formation et de réseautage qui s'adresse particulièrement aux entrepreneurs (PME de moins de dix employés), aux travailleurs autonomes et aux personnes en recherche d'emploi de Brossard et de la Rive-Sud, mais qui est également accessible à l'ensemble de la population.

Le Centre est sous la responsabilité d'une bibliothécaire à temps plein qui relève de la directrice de la bibliothèque. Outre la bibliothécaire, l'équipe est composée d'une technicienne en documentation, d'une coordonnatrice du prêt et de deux commis en bibliothèque qui assument, à temps partiel, différentes tâches : catalogage et commande des documents, pointage des périodiques et abonnements.

En 2009, le Centre soulignait ses 10 ans au service des gens d'affaires par la tenue d'un événement de type 5 à 7 , par le dévoilement de son nouveau logo et par la refonte de son site Internet (<http://centreaffaires.brossard.ca>). 


\section{Les services offerts}

\section{Aide à la recherche}

Le service d'aide à la recherche se présente sous deux formes. La première consiste en un accompagnement dans le cadre d'une recherche : repérage de documents sur le commerce électronique, initiation à l'utilisation d'une base de données, référence à un organisme tel que le Centre local de développement ${ }^{2}$, qui aidera l'entrepreneur à réaliser son plan d'affaires. La deuxième forme d'assistance est la recherche réalisée par le personnel du Centre permettant de trouver, entre autres, les coordonnées d'une entreprise ou d'un fournisseur, des modèles de plan d'affaires ou de plan budgétaire, les perspectives d'emploi dans un secteur donné, une liste de concurrents et des statistiques de tout genre.

\section{Ateliers pratiques pour les PME et travailleurs autonomes}

Le Centre d'affaires.doc offre des ateliers pratiques et spécialisés en lien avec les préoccupations des entrepreneurs et des travailleurs autonomes. Douze ateliers d'une durée de trois heures chacun sont présentés au cours de l'année et abordent des thématiques telles que la fiscalité du travailleur autonome, les outils de présentation, la gestion du temps, le financement d'une entreprise au démarrage, le marketing, le service à la clientèle, la gestion efficace des coûts, l'efficacité des réseaux sociaux et comment attirer et fidéliser la clientèle avec son site Web.

Ces ateliers permettent aux participants d'améliorer leur connaissance du milieu des affaires et d'effectuer du réseautage. Ils permettent également aux gens d'avoir des réponses rapides à leurs questions et les aident à mieux avancer sur le chemin de l'emploi et des affaires. En 2010, près de 170 personnes ont participé aux ateliers du Centre.

\section{Bulletin d'information}

Le Centre publie un bulletin Web mensuel qui présente les activités du Centre, les nouveaux membres, les événements et les concours dans le domaine des affaires, les nouvelles acquisitions et toute autre information susceptible d'intéresser sa clientèle comme, par exemple, un blogue pour les entrepreneurs ou un nouveau service de location d'espaces commerciaux. Près de 400 personnes reçoivent le bulletin par courrier électronique. L'abonnement se fait directement sur le site Web du Centre.

\section{Collections}

La collection du Centre d'affaires.doc est constituée de documents en langues française et anglaise. La priorité est d'abord accordée aux documents québécois et ensuite aux documents canadiens, américains et européens. La clientèle a accès à plus de 5000 documentaires (livres et DVD) sur l'entrepreneurship, le marché du travail, la recherche d'emploi, la formation, les finances personnelles, le marketing, la comptabilité, etc. ; à près de 500 ouvrages de référence qui sont des répertoires d'entreprises, des recueils de statistiques, des publications gouvernementales, des normes, des répertoires scolaires, des dictionnaires spécialisés et de la documentation relative aux subventions et programmes d'aide aux entrepreneurs; et à plus de 50 revues et journaux dont Les Affaires, PME et Investor's Digest of Canada.

Quinze bases de données sont également disponibles, dont certaines en ligne. ICRIQ ${ }^{3}$, Scott's National All, Selectory, Business Source Elite et Weblocal permettent de repérer des entreprises. De l'information sur l'emploi, la carrière et la formation sont disponibles par l'entremise de Webcarrière, Canadian Employment Weekly, CareerAim et Repères-GRICS. Référence RH fournit des informations sur les ressources humaines et CANSIM, des données statistiques. La clientèle a également accès aux bases Eureka, Repère, Canadian Newsstand et Canadian Reference Centre pour la recherche d'articles de revues et de journaux. Ces dernières, avec Selectory, sont les bases de données les plus utilisées par les usagers.

Les ateliers et le bulletin sont les services les plus populaires du Centre parce qu'ils répondent rapidement et efficacement aux questionnements et aux besoins d'information des travailleurs autonomes et des entrepreneurs.

\section{Membership}

Une particularité du Centre d'affaires.doc est le fait que les entreprises de Brossard peuvent devenir membre $\mathrm{du}$ Centre gratuitement. Les entreprises des autres villes peuvent également le devenir, mais au coût de 50 \$ par année. Le Centre comptait en 2010 plus de 90 membres qui bénéficient ainsi de plusieurs privilèges : visibilité corporative sur le site Internet du Centre, dans le bulletin Web et sur le babillard dans la bibliothèque ; réservation de salles de réunion; participation à des événements spéciaux comme le 5 à $7 \mathrm{du} 10^{\mathrm{e}}$ anniversaire ; rabais pour les activités des partenaires; emprunt de documents et accès aux bases de données en ligne. 


\section{Partenariats}

Le Centre d'affaires.doc a établi jusqu'à maintenant 17 partenariats avec des organismes de Brossard et de la Rive-Sud. Parmi les partenaires, on retrouve l'Association communautaire d'emprunt de la Rive-Sud, la Caisse Desjardins de Brossard, le Centre de formation professionnelle Pierre-Dupuy, les Entreprises Jeunesse de la Montérégie, Génération XYZ, la Maison internationale Rive-Sud et le Réseau des femmes d'affaires du Québec, secteur Montérégie.

Les mandats de ces partenaires sont :

- de soutenir et d'encadrer les entrepreneurs, comme c'est le cas pour le Centre local de développement de Longueuil et la cellule de mentorat d'affaires de la Chambre de commerce et d'industrie de la Rive-Sud ;

- d'informer sur le démarrage et le financement d'une entreprise comme, par exemple, les Cercles d'entraides de la Rive-Sud et Femmessor;

- d'aider les gens en recherche d'emploi, en orientation de carrière et en intégration sur le marché du travail, comme c'est le cas pour le Carrefour Jeunesse-Emploi La Pinière, le Centre local d'emploi de Brossard, le Club de recherche d'emploi Châteauguay-Brossard et De soi à l'emploi.

Le Centre et ses partenaires collaborent afin de bonifier leur offre de services respective et maximiser l'utilisation de leurs ressources. Les organismes desservant à peu près la même clientèle, il s'agit également d'éviter de refaire ce qui existe ou est déjà offert ailleurs. En somme, le Centre et ses partenaires sont complémentaires et bénéficient d'une réciprocité dans les services et la visibilité.

Pour les partenaires, la collaboration avec le Centre d'affaires.doc signifie une visibilité corporative sur le site Web du Centre et dans le bulletin Web, une présentation de leurs activités sur le babillard du Centre et la distribution de leurs dépliants dans les locaux du Centre. Les partenaires ont aussi accès aux salles de réunion, d'animation et de formation (avec 18 postes informatiques) de la bibliothèque pour la tenue de leurs activités. Leurs clientèles ont également accès aux formations informatiques (utilisation du clavier,
Tableau 1

Statistiques d'utilisation (2010)

\begin{tabular}{|l|c|}
\hline Nombre de membres & 91 \\
\hline Utilisation des salles de réunion & 67 \\
\hline Abonnements à la liste de diffusion & 402 \\
\hline Collections & \\
- Documentaires & 5000 \\
- Référence & 500 \\
- Journaux et revues & 50 \\
- Bases de données & 15 \\
\hline Emprunts & 7249 \\
\hline
\end{tabular}

de la souris, d'Internet et du courriel) données par le personnel du Centre.

Pour le Centre d'affaires.doc, la collaboration avec les partenaires se traduit par une visibilité corporative sur les sites Web des partenaires, la diffusion du bulletin Web auprès de leur clientèle, la présentation du logo du Centre lors de leurs événements et la présentation du Centre, par la responsable, à leur clientèle. Les partenaires distribuent également les dépliants du Centre dans leurs locaux et animent des ateliers gratuitement.

\section{Conclusion}

Depuis maintenant plus de 10 ans, le Centre d'affaires.doc soutient sa clientèle : en favorisant les contacts, dans le cadre des ateliers, entre les participants pour le réseautage et l'obtention de contrat ; en permettant aux entrepreneurs et aux travailleurs autonomes, toujours dans le cadre des ateliers, de bénéficier de l'expérience des experts pour atteindre le but visé plus rapidement et d'apprendre sur des aspects qu'ils connaissent moins; et en améliorant leurs connaissances, par le biais des collections et du bulletin d'informations, pour obtenir les outils et les compétences nécessaires pour réaliser leurs activités. Le Centre est très sollicité, comme le révèlent les statistiques de fréquentation et d'activités pour l'année 2010 (Tableau 1).

Le Centre entend élargir son offre de services en proposant des initiations à l'emploi et à l'entrepreneurship ainsi qu'en donnant des formations sur la gestion d'un budget pour les adolescents. $\odot$ 